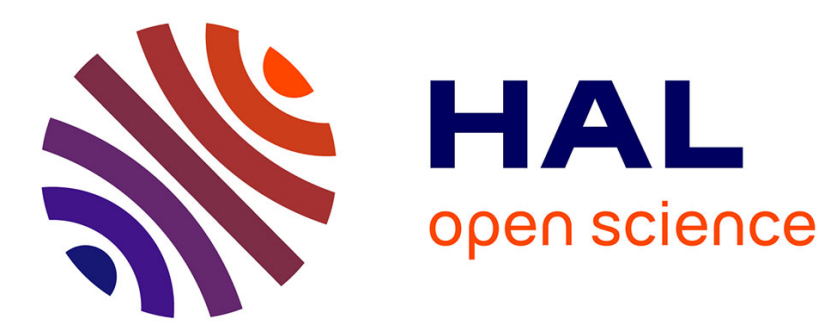

\title{
Analysis of Noisy Digital Contours with Adaptive Tangential Cover
}

Phuc Ngo, Isabelle Debled-Rennesson, Bertrand Kerautret, Hayat Nasser

\section{To cite this version:}

Phuc Ngo, Isabelle Debled-Rennesson, Bertrand Kerautret, Hayat Nasser. Analysis of Noisy Digital Contours with Adaptive Tangential Cover. Journal of Mathematical Imaging and Vision, 2017, 59 (1), pp.123-135. 10.1007/s10851-017-0723-7 . hal-01486969

\section{HAL Id: hal-01486969 \\ https://hal.inria.fr/hal-01486969}

Submitted on 10 Mar 2017

HAL is a multi-disciplinary open access archive for the deposit and dissemination of scientific research documents, whether they are published or not. The documents may come from teaching and research institutions in France or abroad, or from public or private research centers.
L'archive ouverte pluridisciplinaire HAL, est destinée au dépôt et à la diffusion de documents scientifiques de niveau recherche, publiés ou non, émanant des établissements d'enseignement et de recherche français ou étrangers, des laboratoires publics ou privés. 


\title{
Analysis of Noisy Digital Contours with Adaptive Tangential Cover
}

\author{
Phuc Ngo - Isabelle Debled-Rennesson • Bertrand Kerautret - Hayat \\ Nasser
}

Received: date / Accepted: date

\begin{abstract}
The notion of tangential cover, based on maximal segments, is a well-known tool to study the geometrical characteristics of a discrete curve. However, it is not robust to noise, while extracted contours from digital images typically contain noise and this makes the geometric analysis tasks on such contours difficult. To tackle this issue, we investigate in this paper a discrete structure, named Adaptive Tangential Cover (ATC), which is based on the notion of tangential cover and on a local noise estimator. More specifically, the ATC is composed of maximal segments with different widths deduced from the local noise values estimated at each point of the contour. Furthermore, a parameter-free algorithm is also presented to compute ATC. This study leads to the proposal of several applications of ATC on noisy digital contours: dominant point detection, contour length estimator, tangent/normal estimator, detection of convex and concave parts. An extension of ATC to 3D curves is also proposed in this paper. The experimental results demonstrate the efficiency of this new notion.
\end{abstract}

Keywords maximal blurred segment - noise level . geometrical parameters - dominant points - tangent . normal vectors $\cdot$ length contour estimator $\cdot$ contour concave/convexe parts

\section{Introduction}

In shape recognition or shape matching in image processing, the geometric analysis of digital shape contour

P. Ngo, H. Nasser, I. Debled-Rennesson, B. Kerautret

Loria - Campus Scientifique - BP 239 - 54506 Vandoeuvre-ls-

Nancy Tel.: +33-3-83592000

Email: hoai-diem-phuc.ngo@loria.fr is a fundamental task. A common approach is to describe the discrete curves of contours by their lists of points but geometrical information like curvature or tangent does not appear in this description. For more than ten years, in discrete geometry field, the notion of maximal segment has been widely used to describe and to analyze the geometric properties of the contour of digital shapes. Based on the definition of discrete line [1], the sequence of all maximal discrete straight segments (maximal DSS) along a digital contour $C$ is called the tangential cover and can be computed in $O(N)$ time complexity [2] where $N$ is the number of points on the contour. In [3], F. Feschet studies the structure of discrete curves with tangential cover and shows that the tangential cover has the property of being unique and canonical when computed on closed curves. Tangential cover and maximal segments induce numerous discrete geometric estimators (see [4] for a state of the art): length, tangent, curvature estimators, detection of convex or concave parts of a curve, minimum length polygon of a digital contour, detection of the noise level possibly damaging the shape $[5,6]$.

However, as the tangential cover use the rigid arithmetical definition of DSS [1], it is not adapted to noisy digital contours. To deal with this issue, several methods based on non-DSS -often with parameters- were presented as in $[7,8]$. There are likewise other approaches that have been proposed to obtain a better model of tangential cover, adapted to noise. One of them consists in using the notion of maximal blurred segments (MBS) which is an extension of maximal segments with a width parameter $[9,12]$. It was used in several geometric estimators: curvature estimator [12], dominant point detection [13,14], circularity detection, arc and segment decomposition $[15,16]$. Nevertheless, the width parameter needs to be manually adjusted and the method is 
not adaptive to local amount of noise which can appear on real contours.

This paper is an extension of the work proposed in [17]: we present a parameter-free framework to analyze noisy digital shape contours. We use a new notion, named Adaptive Tangential Cover (ATC) An ATC of a digital contour is composed of MBS with appropriate widths, deduced from the noise level detected in the contour. The local noise estimator computes a value at each point of the discrete contour. It permits to determine the widths of MBS composing the ATC. Therefore the algorithm to compute ATC is parameter-free. The proposed framework works with different local noise estimators and, in this paper, we focus on two estimators presented in $[18]$ and in $[5,6]$.

We apply the ATC to extract geometric information of noisy contours. In particular, an extension of dominant point detection algorithm [14] with ATC is proposed to obtain polygonal representations of noisy discrete contours. This leads to the proposal of a length estimator for noisy contours. Furthermore, a tangent estimator, based on the $\lambda$-MST $[19,20]$, is presented, well adapted to noisy contours. By using the slopes of the successive MBS of the ATC, a detector of convex and concave parts of a contour is also proposed. Experimentations and comparisons with other methods show the interest of the proposed framework using ATC.

Moreover we propose a first approach of the notion of 3D ATC, directly deduced from the ATC of 2D curves.

The paper is organized as follows: in section 2, we recall all necessary definitions and results to make the paper self-content. Then, in section 3 , we describe the discrete structure named Adaptive Tangential Cover (ATC), and we illustrate the construction algorithm of ATC. In section 4, applications to analyze and to extract geometric information from noisy contours are presented as well as experimental results and an extension of ATC to 3D curves.

\section{Geometrical tools for discrete curves analysis}

We recall in this section several notions of discrete geometry, very useful in the study of discrete curves. The main ideas of previous works are presented here and we refer the reader to the given references for more details.

\subsection{Maximal blurred segments}

As previously described, the discrete primitives, such as discrete lines [1], blurred segments [9] and maxi- mal blurred segments [12] have been used in numerous works to determine geometrical characteristics of discrete curves.

Definition 1 A discrete line $\mathcal{D}(a, b, \mu, \omega)$, with a main vector $(a, b)$, a lower bound $\mu$ and an arithmetic thickness $\omega$ (with $a, b, \mu$ and $\omega$ being integers such that $\operatorname{gcd}(a, b)=1)$ is the set of integer points $(x, y)$ verifying $\mu \leq a x-b y<\mu+\omega$. Such a line is denoted by $\mathcal{D}(a, b, \mu, \omega)$.

Let us consider $\mathcal{S}$ as a sequence of integer points.

Definition 2 A discrete line $\mathcal{D}(a, b, \mu, \omega)$ is said to be bounding for $\mathcal{S}$ if all points of $\mathcal{S}$ belong to $\mathcal{D}$.

Definition 3 A bounding discrete line $\mathcal{D}(a, b, \mu, \omega)$ of $\mathcal{S}$ is said to be optimal if the value $\frac{\omega-1}{\max (|a|,|b|)}$ is minimal, i.e. if its vertical (or horizontal) distance is equal to the vertical (or horizontal) thickness of the convex hull of $\mathcal{S}$

This definition is illustrated in Fig. 1 and leads to the definition of the blurred segments.

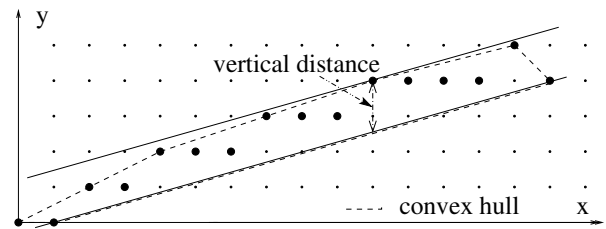

Fig. $1 \mathcal{D}(2,7,-8,11)$, the optimal bounding line of the set of points (vertical distance $=\frac{10}{7}=1.42$ ).

Definition 4 A set $\mathcal{S}$ is a blurred segment of width $\nu$ if its optimal bounding line has a vertical or horizontal distance less than or equal to $\nu$ i.e. if $\frac{\omega-1}{\max (|a|,|b|)} \leq \nu$.

The notion of maximal blurred segment was introduced in [12]. Let $C$ be a discrete curve and $C_{i, j}$ a sequence of points of $C$ indexed from i to j. Let us suppose that the predicate " $C_{i, j}$ is a blurred segment of width $\nu$ " is denoted by $B S(i, j, \nu)$.

Definition $5 C_{i, j}$ is called a maximal blurred segment of width $\nu$ and noted $M B S(i, j, \nu)$ iff $B S(i, j, \nu)$, $\neg B S(i, j+1, \nu)$ and $\neg B S(i-1, j, \nu)$.

The following important property was proved in [12]

Property 1 Let $M B S_{\nu}(C)$ be the set of width $\nu$ maximal blurred segments of the curve $C$. Then, $M B S_{\nu}(C)=\left\{M B S\left(B_{0}, E_{0}, \nu\right), M B S\left(B_{1}, E_{1}, \nu\right), \ldots\right.$, $\left.M B S\left(B_{m-1}, E_{m-1}, \nu\right)\right\}$ and satisfies $B_{0}<B_{1}<\ldots<$ $B_{m-1}$. So we have: $E_{0}<E_{1}<\ldots<E_{m-1}$. 
Deduced from the previous property, an incremental algorithm was proposed in [12] to determine the set of all maximal blurred segments of width $\nu$ of a discrete curve $C$. The main idea is to maintain a blurred segment when a point is added (or removed) to (from) it. The obtained structure for a given width $\nu$ can be considered as an extension of the tangential cover [2] and we name it width $\nu$ tangential cover of $C$. Using the method proposed in [21], such a tangential cover can be computed in $O(n \log n)$. Examples of tangential covers for different widths are given in Fig. 6(c-f) and in Fig. 2.

\subsection{Local noise estimators}

In [5,6], a notion of Meaningful Scale (MS), was designed to locally estimate what is the best scale to analyze a digital contour. This estimation is based on the study of the asymptotic properties of the discrete lengths $L$ (number of grid points) of maximal digital straight segments (maximal DSS, based on definition 1). In particular, it has been shown that the lengths of maximal DSS covering a point $P$ located on the boundary of a $C^{3}$ continuous object should be between $\Omega\left(1 / h^{1 / 3}\right)$ and $O\left(1 / h^{1 / 2}\right)$ if $P$ is located on a strictly concave or convex part and near $O(1 / h)$ elsewhere (where $h$ represents the grid size). This theoretical property defined on finer and finer grid sizes was used by taking the opposite approach with the computation of the maximal segment lengths obtained with coarser and coarser grid sizes (from subsampling). Such a strategy is illustrated in Fig. 2 (a-c) with a source point $P$ and its tangential cover defined from subsampling grid size equals to 2 (Fig. 2 (b)) and 3 (Fig. 2 (c)). From the graph of the maximal DSS mean lengths $L^{i}$ obtained at different scales, the method consists in recognizing the first scale for which the lengths follow the previous theoretical behavior.

As mentioned in $[5,6]$, the multiscale profile can be used to locally detect a scale interval considered as meaningful. From this notion of meaningful scale, a noise level can be deduced and associated to each point of the contour (see Fig. 5). In the following, we called MS estimator this local noise level estimator.

The previous method of meaningful scale detection [5, 6] has been extended to the detection of the Meaningful Thickness (MT) [18]. This method mainly differs by the choice of the blurred segment primitive and by the scale definition which is given by the width parameter of the blurred segment (called thickness in [18]). Such a strategy presents the first advantage to be easier to implement without the need to apply different subsamplings. The length variation of the maximal blurred seg-
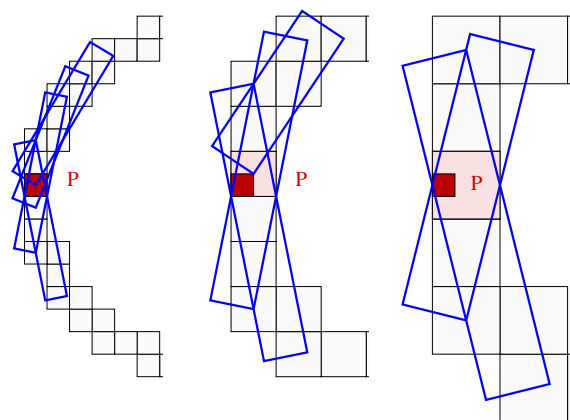

(a) $\overline{L^{1}}=9.25$

(b) $\overline{L^{2}}=6.33$

(c) $\overline{L^{3}}=5.5$
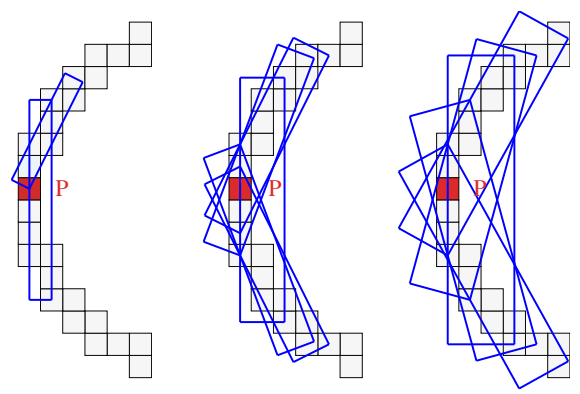

(d) $\overline{\frac{\mathcal{L}^{1}}{1}}=7.18$

(e) $\frac{\overline{\mathcal{L}^{2}}}{2}=4.78$

(f) $\overline{\frac{\mathcal{L}^{3}}{3}}=3.87$

Fig. 2 Images (a-c) illustrate the maximal segments (with the mean $\bar{L}$ of their discrete length) used in the meaningful scale estimation computed by subsampling the initial contour (a). The equivalent blurred segments defined with different widths illustrate the primitives used in the notion of meaningful thickness (d-f). The mean $\overline{\mathcal{L}^{k}}$ of the Euclidean lengths of the boxes bounding the width $k$ blurred segments are given for $3 k$ values.

ments (Euclidean lengths of the bounding boxes of the blurred segments) obtained at different widths appears to follow the equivalent properties for the maximal DSS defined from sub-sampling. Fig. 3 shows the comparison of the length variations obtained with the maximal DSS (b) and with the maximal blurred segments (c). In both cases, the evolution of lengths presents equivalent slopes which are included in the same interval. More formally, if we denote by $t_{i}$ the width of value $i$, a multi-thickness profile $\mathcal{P}_{n}(P)$ of a point $P$ is defined in [18] as the graph

$$
\left(\log \left(t_{i}\right), \log \left(\overline{\mathcal{L}}^{t_{i}} / t_{i}\right)\right)_{i=1, \ldots, n}
$$

with $\overline{\mathcal{L}}^{t_{i}}$, the mean of the Euclidean lengths of the boxes bounding the width $t_{i}$ blurred segments. The following conjecture has been experimentally checked.

Conjecture 1 (Multi-thickness). The plots of the lengths $\mathcal{L}_{j}^{t_{i}} / t_{i}$ in log-scale are approximately affine with negative slopes $s$ located between $-\frac{1}{2}$ and $-\frac{1}{3}$ for a curved part and around -1 for a flat part. 


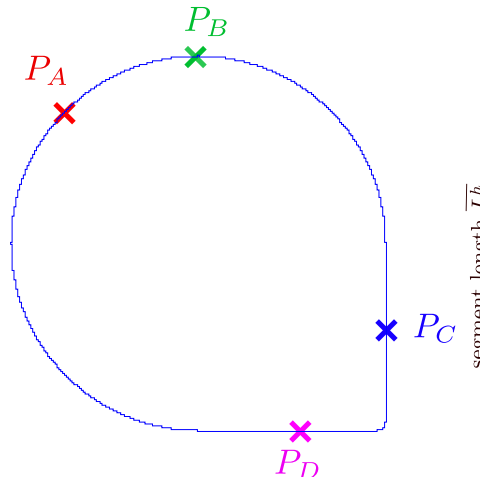

(a) curve

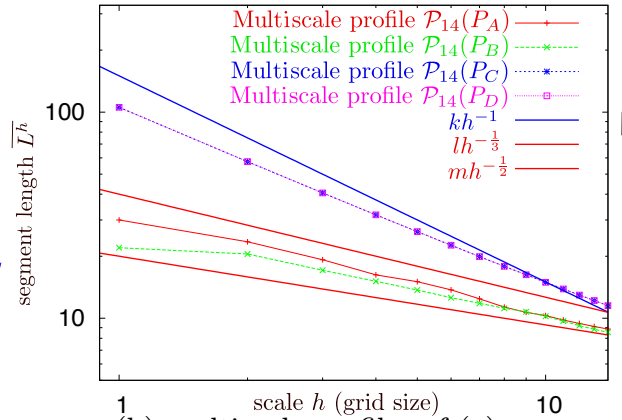

(b) multiscale profiles of (a)

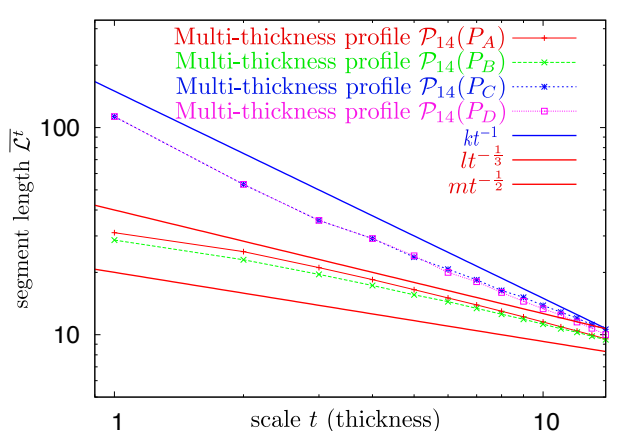

(c) multi-thickness profiles of (a)

Fig. 3 Comparison between multiscale (b) and multi-thickness (c) profiles on different types of points defined on a shape (a) containing curved $\left(P_{A}, P_{B}\right)$ and flat $\left(P_{C}, P_{D}\right)$ parts.

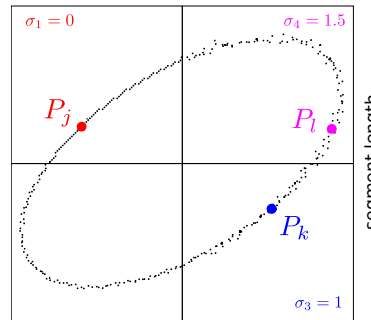

(a)

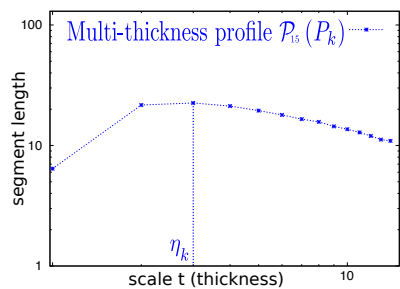

(c)

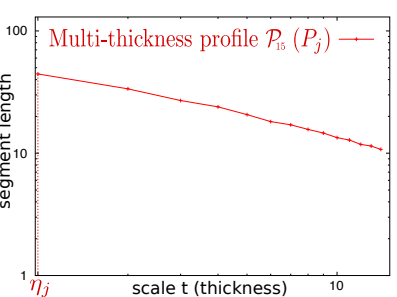

(b)

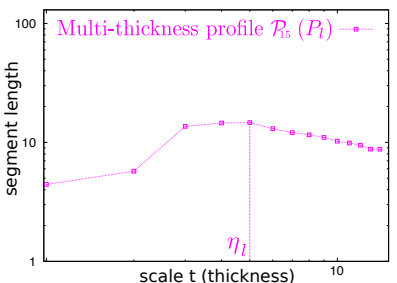

(d)
Fig. 4 Multi-thickness profiles (b-d) obtained on different points: $P_{j}$ with no noise (graph (b)), with low noise $\left(P_{k}\right.$, graph $(\mathrm{c}))$ and important noise $\left(P_{l}\right.$, graph $\left.(\mathrm{d})\right)$. The meaningful thickness values $\eta_{j}, \eta_{k}$ and $\eta_{l}$ are represented on each multi-thickness profile $\mathcal{P}_{15}$.

Such a profile is illustrated on Fig. $4(\mathrm{a}, \mathrm{b})$ where a multi-thickness profile is given on a point located on a contour part presenting no noise.

From $\mathcal{P}_{n}\left(P_{j}\right)=\left(X_{i}, Y_{i}\right)_{i=1, \ldots, n}$, the multi-thickness profile of $P_{j}$, the meaningful thickness is defined as a pair $\left(i_{1}, i_{2}\right), 1 \leq i_{1}<i_{2} \leq n$, such that for all $i$, $i_{1} \leq i<i_{2}, \frac{Y_{i+1}-\bar{Y}_{i}}{X_{i+1}-X_{i}} \leq T_{m}$, and the property is not true for $i_{1}-1$ and $i_{2}$. As suggested in [18], the value of the parameter $T_{m}$ is set to 0 . In the following, we will denote by $\eta_{j}$ the value $i_{1}$ corresponding to the first meaningful thickness $\left(i_{1}, i_{2}\right)$ of a point $P_{j} . \eta_{j}$ is named meaningful thickness value (MT value) and permits to detect the noise level at each point $P_{j}$ of a curve. This local noise level estimator is called MT estimator.

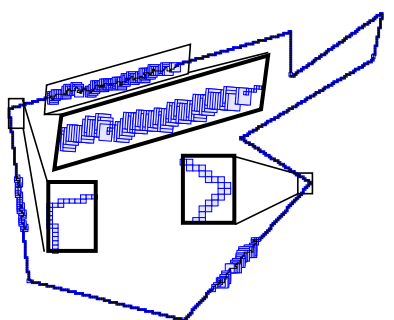

(a)

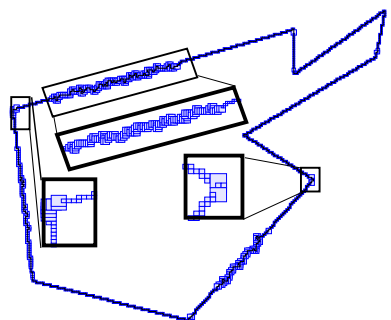

(b)
Fig. 5 Comparison between values obtained with MS estimator (a) and MT estimator (b). The size of the blue boxes represents for each pixel the MS or MT value.

Fig. 4 illustrates the meaningful thickness values obtained for different points $P_{j}, P_{k}$ and $P_{l}$ which present respectively the following values: $\eta_{j}=1, \eta_{k}=3$ and $\eta_{l}=5$. Another illustration of the values obtained with MT estimator is proposed in Fig. 6(b). Fig. 5 shows the comparison between the noise level values obtained with MS estimator and with MT estimator. The MT estimator shows slightly more sensitivity for the corners but has the advantage to be able to process non integer coordinate contours.

A local noise estimator is used in the next section to define an adaptive tangential cover by taking into account the amount of noise on the curve.

\section{Adaptive tangential cover}

The tangential covers applied for dominant point [14] and arc/circle detection [15] use mostly mono-width value (ie single width), denoted by $\nu$. Such a parameter $\nu$ allows to take into account the amount of noise present in digital contours. This method has two drawbacks. Firstly, the value of $\nu$ is manually adjusted in 
order to obtain a relevant approximating polygon of the contours w.r.t. the noise. Secondly, the noise appearing along the contour can be random. In other words, different noise levels can be present along the contours. Fig. 6(b) illustrates the different noise levels detected by the MT estimator.

Thus, using mono-width value for tangential covers is inadequate in case of noisy curves.

To overcome these issues, we present the definition of adaptive tangential cover which is a tangential cover with different width values. To this end, we first introduce the notion of inclusion between two MBS.

Definition 6 Let $C$ be a discrete curve and $M B S_{i}=$ $M B S\left(B_{i}, E_{i},.\right), M B S_{j}=M B S\left(B_{j}, E_{j},.\right)$ with $i \neq j$ be two distinct maximal blurred segments on $C . M B S_{j}$ is said to be included in $M B S_{i}$ if $B_{i} \leq B_{j}$ and $E_{i} \geq$ $E_{j}$, and noted by $M B S_{j} \subseteq M B S_{i}$.

Definition 7 Let $M B S(C)$ be a set of maximal blurred segment of a discrete curve $C . M B S_{i}=M B S\left(B_{i}, E_{i},.\right)$ $\in M B S(C)$ is said largest if for all $M B S_{j} \in M B S(C)$ with $i \neq j, M B S_{j} \nsubseteq M B S_{i}$.

Definition 8 Let $C=\left(C_{i}\right)_{0 \leq i \leq n-1}$ be a discrete curve. Let $\eta=\left(\eta_{i}\right)_{0 \leq i \leq n-1}$ be the vector of noise levels associated to each $C_{i}$ of $C$, obtained with a noise level estimator $\mathcal{E}$. Let $M B S(C)=\left\{M B S_{\nu_{k}}(C)\right\}$ be the sets of MBS for the different values $\nu_{k}$ in $\eta$. An adaptive tangential cover associated to $\mathcal{E}\left(A T C_{\mathcal{E}}\right)$ of $C$ is defined as the set of the largest MBS of $\left\{M B S_{j}=\right.$ $M B S\left(B_{j}, E_{j}, \nu_{k}\right) \in M B S(C) \mid \nu_{k}=\max \left\{\eta_{t} \mid t \in\right.$ $\left.\llbracket B_{j}, E_{j} \rrbracket\right\}$.

A local noise level estimator $\mathcal{E}$ is thus integrated in the construction of $A T C_{\mathcal{E}}$ to provide the information of noise along the contour. More precisely, the $A T C_{\mathcal{E}}$ contains the MBS with width values varying in function of the perturbations obtained by the noise level values from $\mathcal{E}$. Since the noise levels are different along the contour curve, accordingly, the obtained $A T C_{\mathcal{E}}$ has the MBS with bigger width values at noisy zones, and with smaller width values in zones with less or no noise (see Fig. 6(h)). Furthermore, this framework is parameterfree.

The method for computing $A T C_{\mathcal{E}}$ is described in Algorithm 1. This algorithm is divided into two steps: (1) labelling the point with the values from the noise estimator $\mathcal{E}$, and (2) building the $A T C_{\mathcal{E}}$ of the curve from the labels previously obtained.

More precisely, the algorithm is initialized with an empty $A T C_{\mathcal{E}}$ and the labels associated to each point are the same as the noise level values (Lines 2-3).

In the first step (Lines 4-8), the tangential covers with widths corresponding to all different noise levels

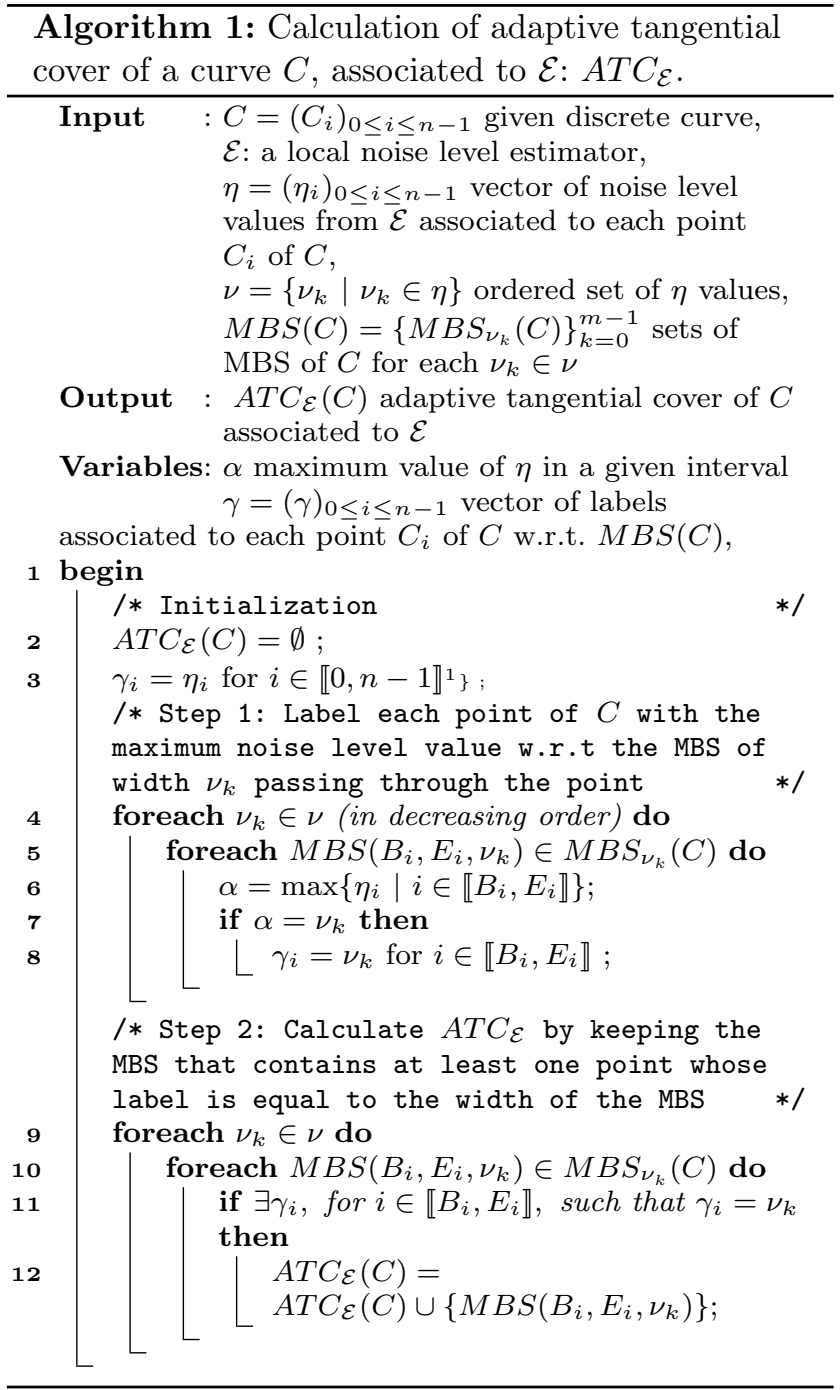

are considered in order to find the label of each point. At each level $\nu_{k}$, the label of a point is updated to $\nu_{k}$ if the MBS passing through the point has the maximal noise level value being equal to $\nu_{k}$. It should be noted that the number of noise levels overall the contour is much smaller than the number of points on the contour. Thus, the number of considered tangential covers is often small. Then, in the second step (Lines 9-12), the $A T C_{\mathcal{E}}$ is composed of the MBS with widths being the label associated to points constituting the MBS.

As stated in section 2.2, the MT estimator allows to locally estimate the noise level at each point of a discrete contour. To illustrate the notion of ATC, we use the MT estimator as noise level estimator and we note the adaptive tangential cover associated to meaningful thickness $\left(A T C_{M T}\right)$. An illustration of the algorithm is given in Fig. 6 .

\footnotetext{
$1 \llbracket a, b \rrbracket$ indicates all integers between $\mathrm{a}$ and $\mathrm{b}$.
} 


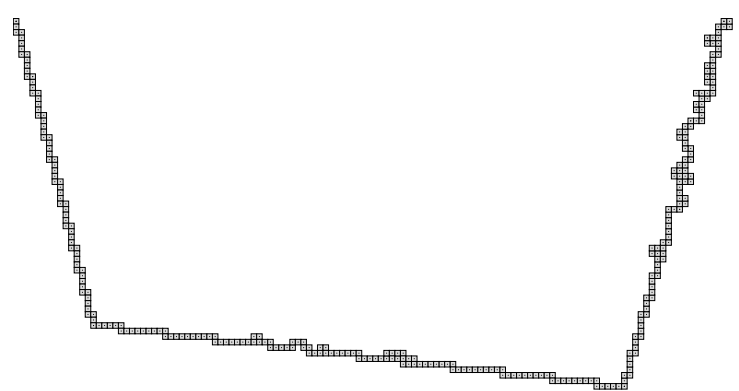

(a) Input discrete curve

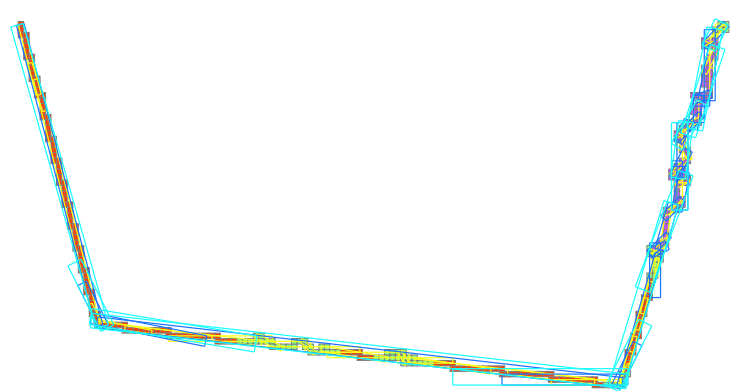

(c) Tangential covers of width $\nu_{k}=1,2,3$

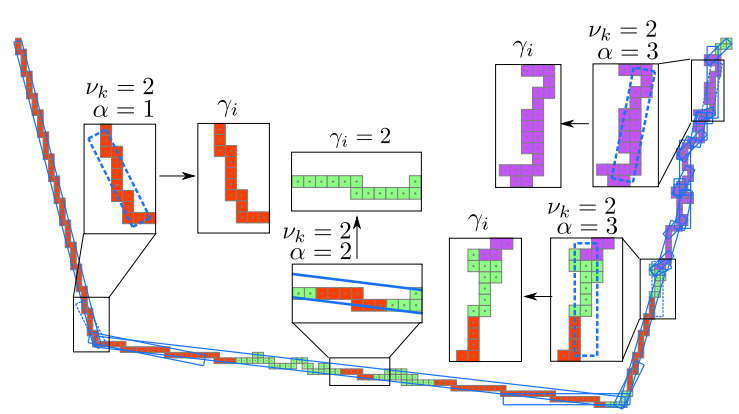

(e) Labeling points with width $\nu_{k}=2$

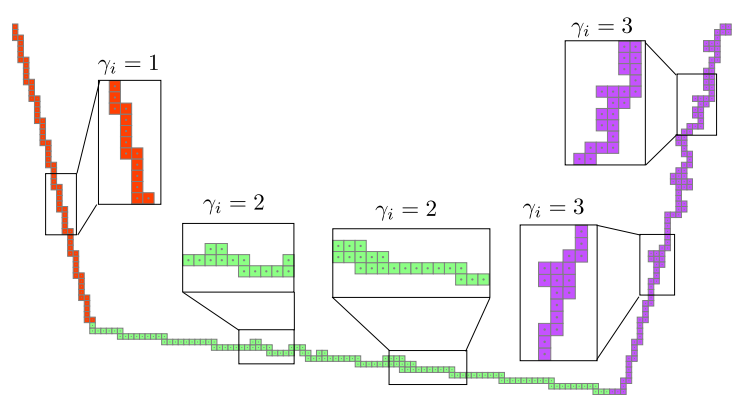

(g) Point's label

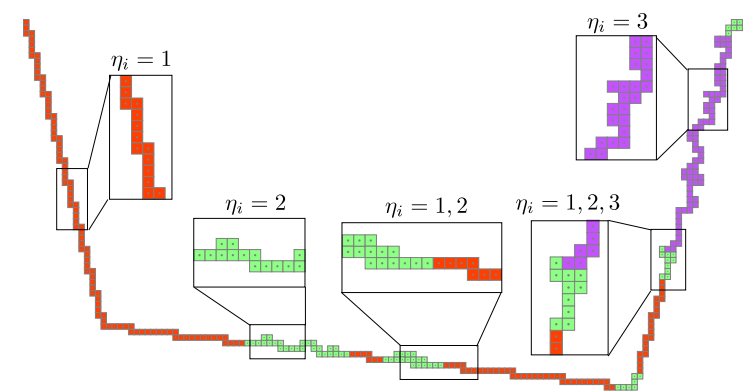

(b) Meaningful thickness $\eta=\{1,2,3\}$

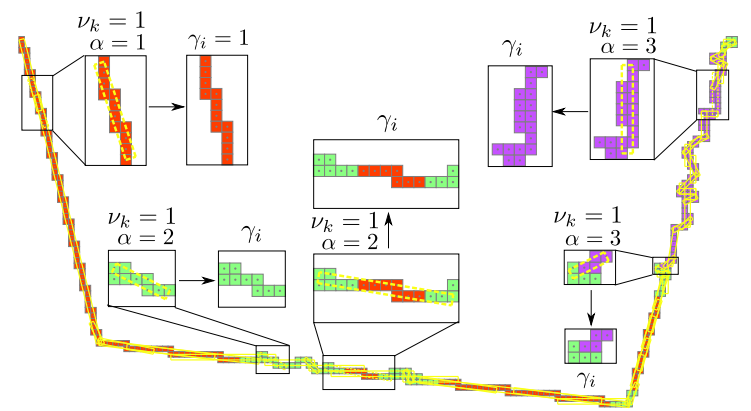

(d) Labeling points with width $\nu_{k}=1$

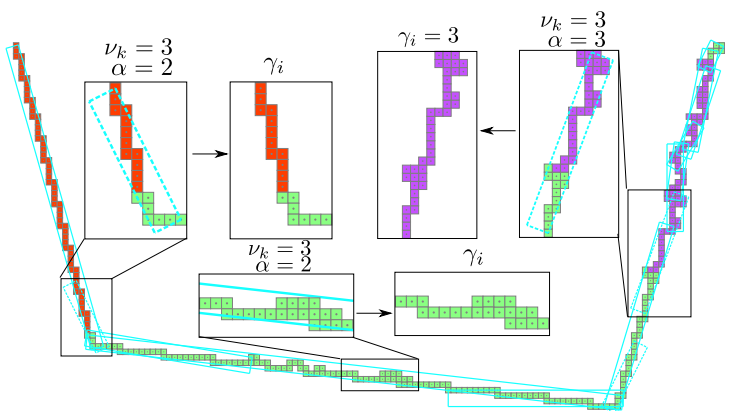

(f) Labeling points with width $\nu_{k}=3$

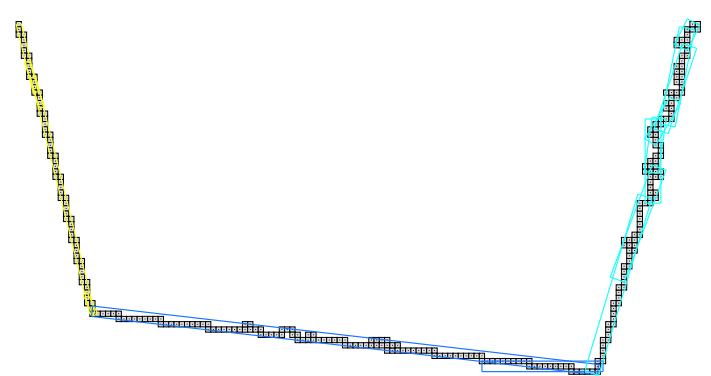

(h) Adaptive tangential cover

Fig. 6 Illustration of Algorithm 1 with $\mathcal{E}=$ MT estimator. (a) Input discrete curve $C$. (b) Noise levels at each point $C_{i}$ of $C$ detected by the MT estimator; the red, green and violet points correspond to the meaningful thickness $\eta_{i}$ of values 1,2 and 3 respectively. The label of each point $C_{i}$ is initialized by its corresponding $\eta_{i}$. (c) Tangential covers of three different widths $\nu_{k}=1,2$ and 3 in yellow, blue and cyan. (d) (e) and (f) Labeling all points $C_{i}$ of $C$ in function of its meaningful thickness values and the tangent covers of widths 1,2 and 3 respectively; The label $\gamma_{i}$ of each point $C_{i}$ is updated to $\nu_{k}$ if the maximal meaningful thickness, namely $\alpha$, of points that belong to the $M B S\left(B_{i}, E_{i}, \nu_{k}\right)$ passing by $C_{i}$ is equal to $\nu_{k}$, and stayed as $\gamma_{i}$ otherwise. (g) Label $\gamma_{i}$ associated to each point of the considering curve. (h) Adaptive tangential cover obtained from the tangential covers and the labels of points. 


\section{Applications}

In this section we present some applications of ATC to dominant point detection, geometric estimators (length and tangent), convex and concave part detector. The experiments are performed on the contours of Fig. 7 obtained by using the extraction of connected region boundary algorithm [22] and the local noise level estimator is the MT estimator. In particular, different noise levels are presented on these contours for the purpose of showing the efficiency of $A T C_{M T}$ in case of non-uniform noise (see Tab. 1). The $A T C_{M T}$ associated to the extracted contour of these images are shown in Fig. 9.

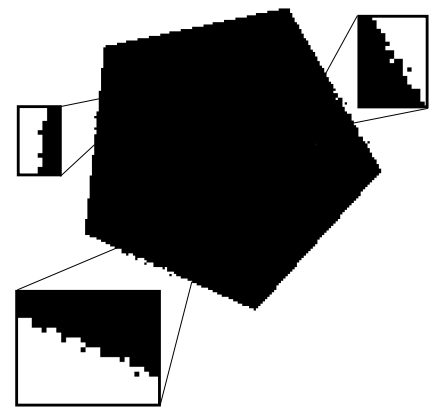

(a)

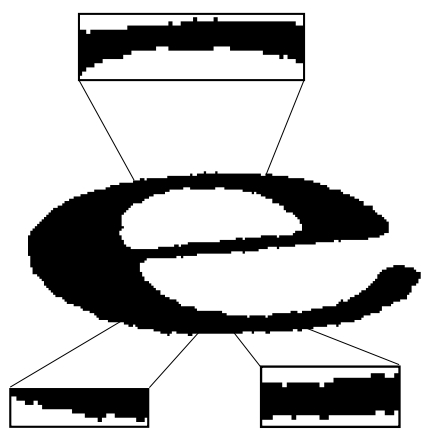

(c)

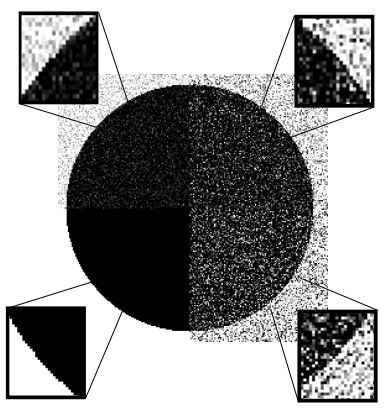

(b)

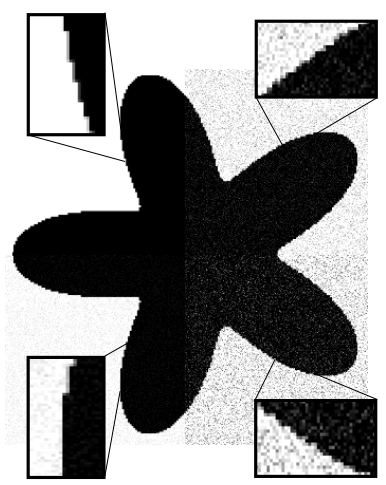

(d)
Fig. 7 Series of input images. (a) (resp. (c)) contains manual (resp. natural) noisy areas (images given in [5]) and (b,d) are obtained by using the Kanugo [23] degradation document model implemented in the imgAddNoise program of the $D G$ talTools project [24].

\subsection{Dominant point detection}

Tangential covers, as stated previously, are involved in applications of dominant point detection $[14,13]$. The previous approaches use tangential covers composed of maximal blurred segments with a constant width along the curve. In general, this parameter needs to be manually adjusted to obtain a good result of detection al-

\begin{tabular}{|l|c|c|c|c|c|c|}
\hline \multirow{2}{*}{ Contour } & \multicolumn{7}{|c|}{ Noise levels } \\
\cline { 2 - 7 } & 1 & 1.5 & 2 & 2.5 & 3 & 3.5 \\
\hline \hline Fig. 7 (a) & $\mathrm{x}$ & $\mathrm{x}$ & $\mathrm{x}$ & & & \\
\hline Fig. 7 (b) & $\mathrm{x}$ & $\mathrm{x}$ & $\mathrm{x}$ & $\mathrm{x}$ & & \\
\hline Fig. 7 (c) & $\mathrm{x}$ & $\mathrm{x}$ & $\mathrm{x}$ & $\mathrm{x}$ & $\mathrm{x}$ & $\mathrm{x}$ \\
\hline Fig. 7 (d) & $\mathrm{x}$ & $\mathrm{x}$ & $\mathrm{x}$ & $\mathrm{x}$ & $\mathrm{x}$ & \\
\hline
\end{tabular}

Table 1 Different noise levels detected by MT estimator on input images in Fig. 7.

gorithm. Therefore, such approaches are not adaptive to discrete contours with irregular noise.

In this section, we present a dominant point detection algorithm using $A T C_{M T}$. The reason is twofold: (1) the $A T C_{M T}$ takes into account the amount of noise on the curve and thus allows a better model of curve segmentation, and (2) the algorithm for computing $A T C_{M T}$ is parameter-free.

We recall hereafter the main idea of the dominant point detection algorithm [14]. Firstly, the algorithm finds the candidates as dominant points which are located in the smallest common zone induced by successive maximal blurred segments [13]. Then, the dominant point of each common zone is identified as the point having the smallest angle with the two extremities of the left and right of the maximal blurred segments composing the zone. This is illustrated in Fig 8.

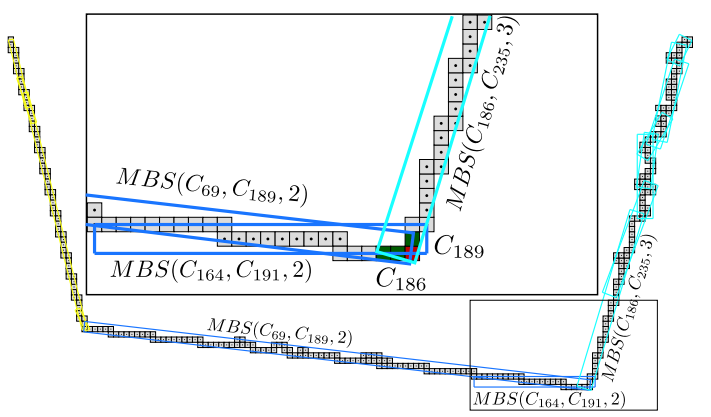

Fig. 8 Illustration of the dominant point detection algorithm with the adaptive tangential cover obtained by Algorithm 1 in Fig. 6. Considering the maximal blurred segments $M B S\left(C_{69}, C_{189}, 2\right), M B S\left(C_{164}, C_{191}, 2\right)$ and $M B S\left(C_{186}, C_{235}, 3\right)$, the common zone determined by these three segments contains four points: $C_{186}, C_{187}, C_{188}$ and $C_{189}$ (green and red points in the zoom). The left and right extremities of the common zone are $C_{69}$ and $C_{235}$ respectively. The angles between each point in the common zone and the two extremities are respectively $102.7^{\circ}, 101.2^{\circ}$, $99.7^{\circ}$ and $100.8^{\circ}$. The dominant point is the point having the smallest angle measure, i.e., $C_{188}$ (red point in the zoom).

Fig. 10 shows the results of dominant point detection using $A T C_{M T}$. 


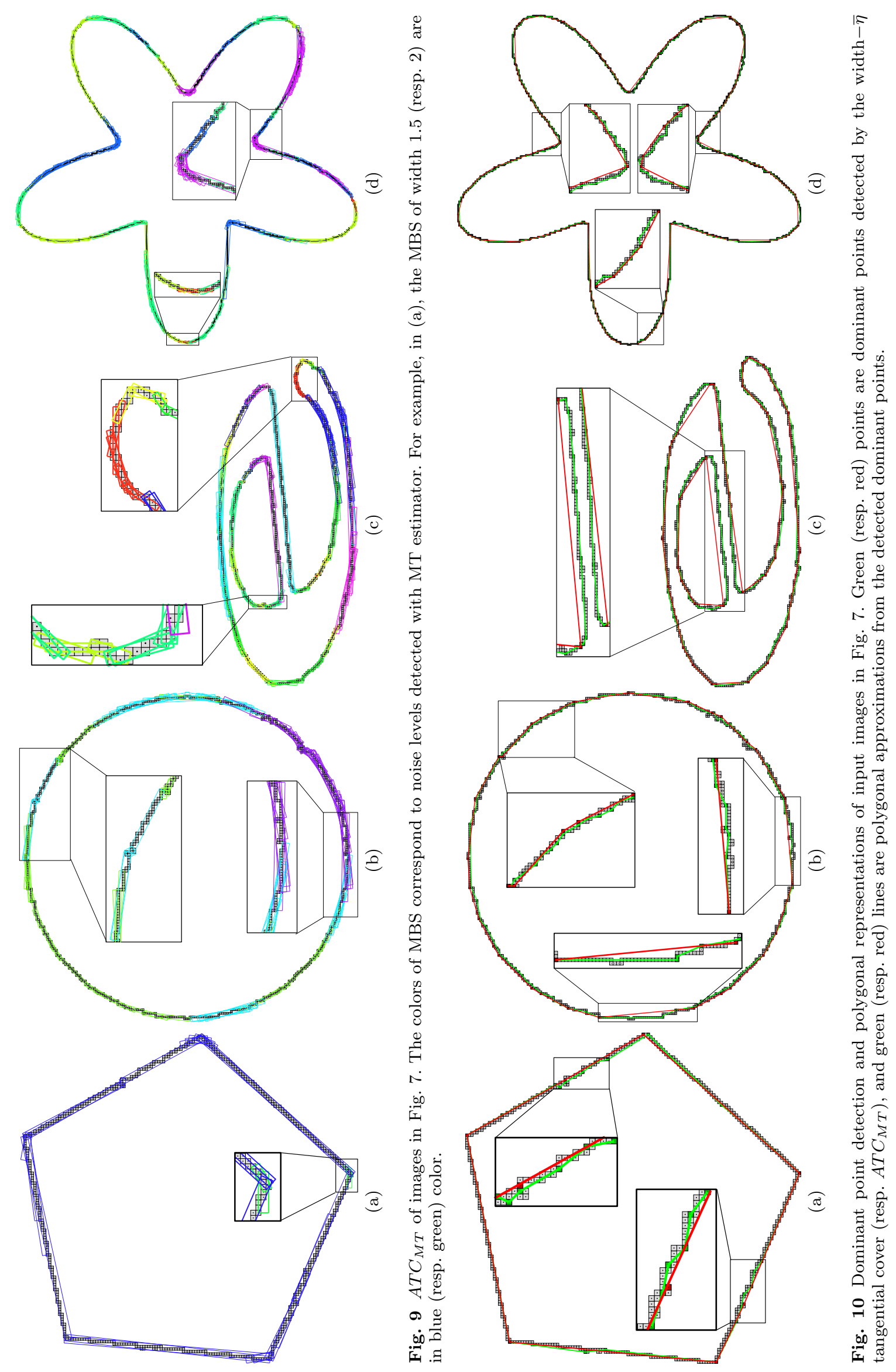


In order to compare the current parameter-free method, we consider in our experiments the mean tangential cover with MBS of width- $\bar{\eta}$ equals to the average of the obtained meaningful thicknesses at each point of the studied curve used in [15].

It can be seen in Fig. 10 that using the mean tangential cover is not always a relevant strategy, particularly in the high noisy zones of curves. This is due to the fact that the width- $\bar{\eta}$ parameter could not capture the local noise on curve, contrary to the $A T C_{M T}$ method (see Fig.10 (a), (d)). In the zoom zones, we observe that the dominant point detected by $A T C_{M T}$ method fits better the corners, whereas the mean method induces a decomposition very close to the studied curve and detects more dominant points. In other words, in the curved zones, the $A T C_{M T}$ method simplifies the representation of the curve.

\subsection{Geometric estimators}

Most of the geometric estimators have a high sensitivity to the noise perturbing digital contours. The $A T C_{M T}$ is composed of MBS with appropriate widths, deduced from the meaningful thicknesses, permits to obtain the three following parameter-free geometric estimators taking into account the level of noise detected in the studied contour.

\subsubsection{Contour length estimator}

An estimator of the contour length is derived naturally from the dominant points obtained with the method presented in the previous section 4.1. The sum of Euclidean distances between each pair of consecutive dominant points on the contour provides an estimation of the length of the studied contour. The $A T C_{M T}$ approach permits to reduce the effects of irregular noise on the contour.

Tab. 2 shows the experimental results using $A T C_{M T}$ and width $\nu$-tangential cover with $\nu=1,2,3$ and 4 [13].

Note that other contour length estimators could be adapted with the proposed adaptive tangential cover in particular by integrating normal vector as described in [25]. Such a possibility could be done by exploiting for instance the $\lambda$-MST estimator described in the next section.

\subsubsection{Tangent estimator based on $\lambda$-MST}

The $A T C_{M T}$ can also be used to improve the precision of existing tangent estimators in presence of noise. In particular, we focus on a multi-grid convergent tangent estimator called the $\lambda$-MST $[19,20]$. This estimator is based on the tangential cover of maximal straight segment and can handle noise by using the blurred segment primitive. More precisely, the tangent estimation is obtained by defining a pencil of maximal blurred segments $\mathscr{P}(k)=\left\{M B S_{i}=\operatorname{MBS}\left(B_{i}, E_{i},.\right)\right.$, with $\left.B_{i} \leq k \leq E_{i}\right\}$ and from a mapping function $\lambda$ defined from $[0,1]$ to $\mathbb{R}^{+}$. From this pencil, a notion of eccentricity was introduced in order to distribute weights on all the segments covering the considered point. More formally, the eccentricity was defined as [20]:

$$
e_{i}(k)=\left\{\begin{array}{cc}
\left\|C_{k}-C_{E_{i}}\right\|_{1} / L_{i} & \text { if } i \in \mathscr{P}(k) \\
0 \quad & \text { otherwise }
\end{array}\right.
$$

The tangent direction $\widehat{\theta}(k)$ of a contour point $C_{k}$ is then computed by: $\frac{\sum_{i \in \mathscr{P}(k)} \lambda\left(e_{i}(k)\right) \theta_{i}}{\sum_{i \in \mathscr{P}(k)} \lambda\left(e_{i}(k)\right)}$. Note that for the experiments presented in this paper we have used the following lambda function $\lambda=64\left(-x^{6}+3 x^{5}-3 x^{4}+x^{3}\right)$.

This estimator can naturally be adapted by including the ATC in the definition of the pencil $\mathscr{P}$ which can contain a set of MBS of different widths. The Fig. 11 illustrates the main idea of this $A T C_{M T} \lambda$-MST estimator. From a given point $C_{1320}$ of a noisy digital contour, three samples of MBS covering it are displayed in light gray $\left(M B S_{0}, M B S_{5}\right.$ and $M B S_{8}$ of $\left.\mathscr{P}(1320)\right)$. From the relative position of $C_{1320}$ according to the index $E_{i}$, we are able to compute the eccentricity of each MBS (see Eq. 1) and we can then deduce the weights from the $\lambda$ function. Finally, the tangent direction $\widehat{\theta}(k)$ can be computed from the different values of $\theta_{i}$ with their associated weights.

The comparisons of Tab. 3 were performed by computing the mean of the absolute error defined by the tangent angle deviation between the estimated and the ground-truth tangents (deduced from the nearest point of the reference shape). The percentage of points having small errors less than 0.1 is also computed to highlight the amount of tangent vectors estimated with precision (denoted as $P_{<0.1}$ ).

Fig. 12 shows the experimental results of the $A T C_{M T}$ $\lambda$-MST tangent estimator with the display of the normal vectors. Tab. 3 presents the comparisons of this estimator with the $\lambda$-MST estimator defined by the $\nu$-tangent covers with $\nu=1,2,3,4$ and the MSMST method proposed by Kerautret and Lachaud in [5]. The $M S M S T$ method, simply consists by assigning, for each point $p$, the $\lambda$-MST tangent value computed from the contour given at the scale defined by the meaning scale of $p$. The $A T C_{M T} \lambda$-MST tangent estimator was also compared with two other digital estimators which are not based on the recognition of discrete straight segments. The first one is the estimator based on the Bi- 


\begin{tabular}{|l|c|c|c|c|c|c|}
\hline \multirow{2}{*}{ Contour } & \multirow{2}{*}{ Ground-truth } & \multicolumn{5}{|c|}{ Estimated length } \\
\cline { 3 - 7 } & & $A T C_{M T}$ & 1-TC & 2-TC & 3-TC & 4-TC \\
\hline \hline Fig. 7 (a) & 411.4497 & 411.594 & 414.416 & 412.046 & 415.639 & 417.09 \\
\hline Fig. 7 (b) & 565.4867 & 565.419 & 573.874 & 568.044 & 563.393 & 558.178 \\
\hline
\end{tabular}

Table 2 Contour length estimator of input images in Fig. 7.

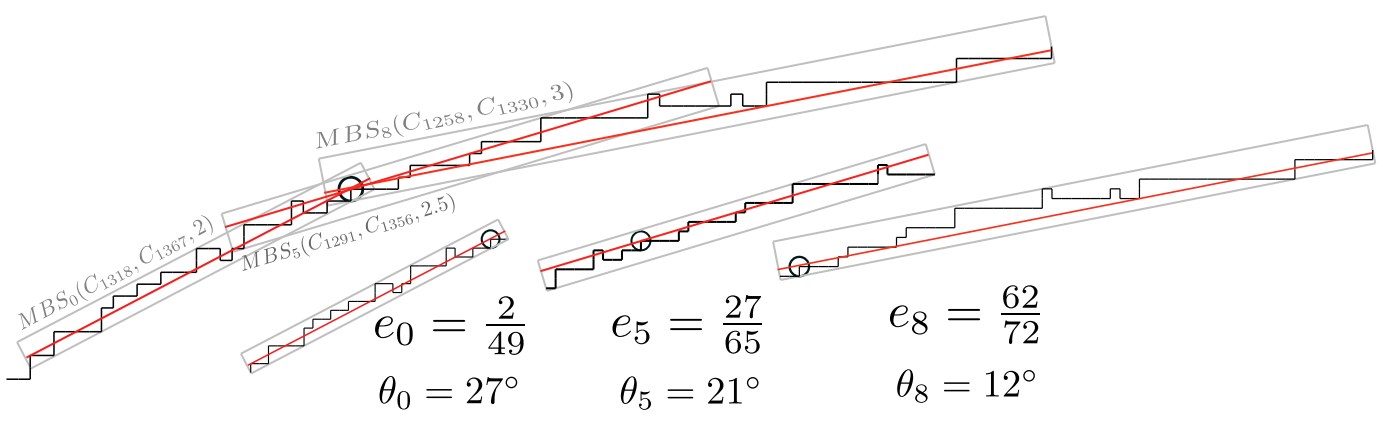

Fig. 11 Illustration of the $\lambda$-MST estimator principle defined from the adaptive tangential cover. A selection of three maximal blurred segments extracted from the pencil $\mathscr{P}(1320)$ are displayed in gray. For each segment, the eccentricity is given with the associated tangent angle.

nomial Convolution $(B C)[7]$ and the second one is the Voronoi Covariance Measure $(V C M)$ based estimator [8] (see Tab. 3). Both methods use parameters which are indicated as indexes of the method names in Tab. 3. The experiments were obtained from the DGtal library [29] and from the DGtalTools [24] implementation.

These results show that our proposed combination of the $\lambda$-MST estimator using $A T C_{M T}$ improves globally the stability of the estimator. It locally preserves the polygonal discontinuities when no noise occurs and it smoothes the tangent directions when some noisy variations appear (even if some noise can locally remain due to the limits of the MT estimator). As shown in Tab. 3 (a) the $A T C_{M T} \lambda$-MST estimator applied on a polygonal shape shows the second best results after the $M S M S T$ estimator which uses the meaningful scale detection (that gives better noise detection than the meaningful thickness on this shape). For the circle the result appears also better than the 1,2,3-TC estimator. The 4-TC estimator, the $V C M_{20,5}$ and $B C$ estimators give better results than the $A T C_{M T} \lambda$-MST or $M S M S T$ estimator but these configurations are not completely significant since the circular shape is not too much deformed by a too large smoothing parameter.

\subsubsection{Convex/concave part detector}

The convexity of digital shape contours is defined and studied in $[26,4]$. A digital shape $O$ (subset of $\mathbb{Z}^{2}$ ) is said digitally convex iff $O$ is 4 -connected and the Gauss digitization of the convex hull of $O$ is equal to $O$.

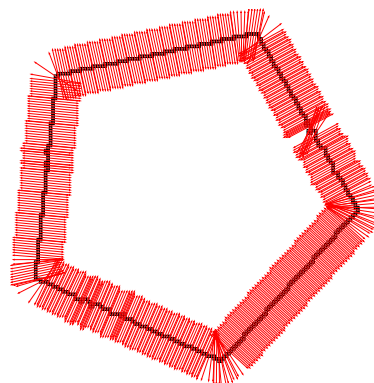

(a)

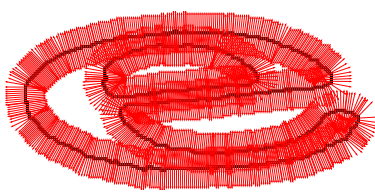

(c)

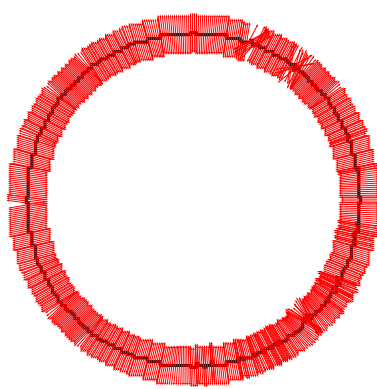

(b)

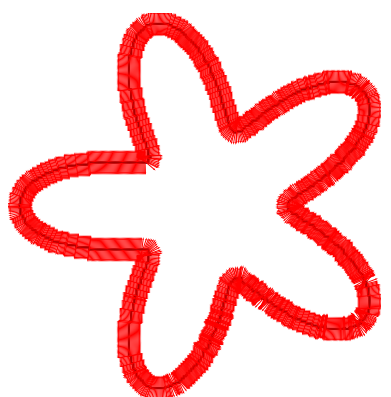

(d)
Fig. 12 Results of $A T C_{M T} \lambda$-MST tangent estimator displayed as normal vectors on the source shape extracted from input images of Fig. 7.

Global convexity information can be deduced from the maximal segments (based on DSS) of the studied contour. Moreover, the convex and concave parts of the shape contour are detected from the successive maximal segments of the tangential cover of the contour. In [26], 


\begin{tabular}{|c|c|c|c|c|c|c|c|c|c|c|c|c|}
\hline \multirow{2}{*}{$\begin{array}{l}\tilde{z} \\
0 \\
\tilde{0} \\
\tilde{O} \\
\end{array}$} & \multirow{2}{*}{ Absolute error } & \multicolumn{11}{|c|}{ Methods } \\
\hline & & $A T C_{M T}$ & 1-TC & $2-\mathrm{TC}$ & $3-\mathrm{TC}$ & 4-TC & MSMST & $V C M_{5,2}$ & $V C M_{10,3}$ & $V C M_{20,5}$ & $B C_{1}$ & $B C_{4}$ \\
\hline$\overbrace{N}^{\overparen{E}}$ & mean & 0.065 & 0.11 & 0.065 & 0.071 & 0.084 & 0.02 & 0.097 & 0.068 & 0.066 & 0.074 & 0.063 \\
\hline$\underset{10}{i 0}$ & $P_{<0.1}$ & 82.1 & 40.4 & 77.9 & 79.5 & 75.5 & 94 & 36.7 & 60.1 & 77.1 & 74.9 & 78.8 \\
\hline$\overbrace{1}^{2}$ & mean & 0.072 & 0.283 & 0.107 & 0.081 & 0.061 & 0.089 & 0.129 & 0.079 & 0.052 & 0.049 & 0.05 \\
\hline$\dot{0}$ & $P_{<0.1}$ & 83 & 27.1 & 72 & 80 & 86.8 & 74.8 & 47.5 & 71.3 & 88.5 & 96.3 & 93.4 \\
\hline
\end{tabular}

Table 3 Errors of tangent estimator of input images in Fig. 7.

it is proved that the contour of a polyomino is digitally convex iff the directions of its maximal segments (based on DSS) are monotonous.

The detector of convex and concave parts on a digital shape contour consists in detecting the sequence of successive maximal segments on the tangential cover whose slopes are monotonous. The maximal segments, whose slope directions are increasing on one side and decreasing on the other side, are transitional areas and they cut the contour of a digital shape into convex and concave parts.

The $A T C_{M T}$ is an extension of the notion of tangential cover with maximal blurred segments (MBS) and we use the same strategy by considering the successive slope directions of MBS. We have tested on several examples the behaviour of the obtained sequence of MBS. For weakly noisy digital contour, the slope directions of MBS in ATC well localize the convex and concave parts of the shape (see Fig 13(c)). For more noisy digital contours, the transitional areas are larger but convex and concave parts are also detected (see Fig. 13(a,d)). In some cases, if noise is irregularly distributed, small perturbations in slope direction induce false detections of convexity or concavity on small parts of the contour (see Fig. 13(b)).

\subsection{Extension of ATC in 3D}

In this section, we propose an extension of ATC in 3D space, namely $3 D A T C$. The main idea is to use the $2 \mathrm{D}$ projections onto the base planes of the $3 \mathrm{D}$ input digital curve. Hereafter, we assume that the 3D input curve has at least two valid ${ }^{2}$ projections. Without loss of generality, we can suppose the valid projections are on $(O, x, y)$ and $(O, x, z)$.

The notion of $3 \mathrm{D}$ discrete line [27] is defined as follows:

\footnotetext{
2 valid in the sense of there are no two points of $3 \mathrm{D}$ digital curve having the same projection onto a $2 \mathrm{D}$ plane.
}

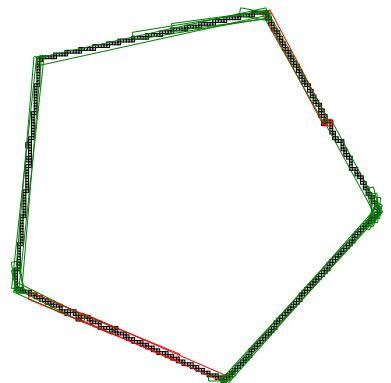

(a)

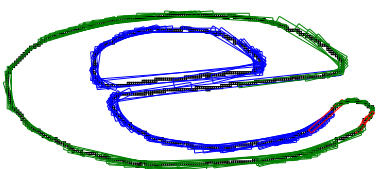

(c)

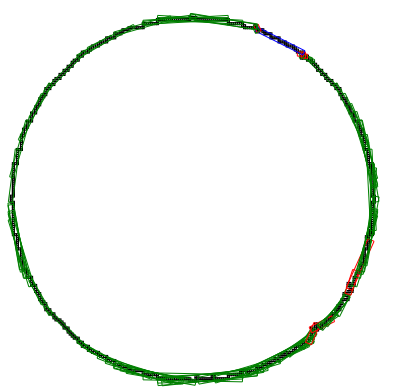

(b)

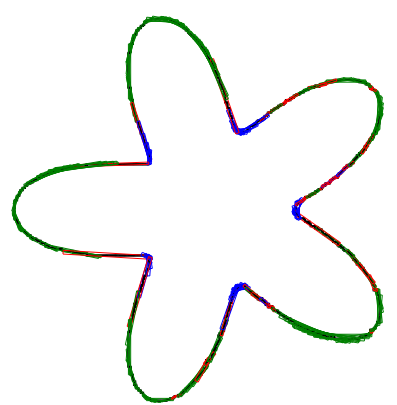

(d)
Fig. 13 Convex and concave part detector of input images in Fig. 7 . The segments in green and blue denote the convex and concave parts respectively. While, the red segments denote a transition between convex and concave parts.

Definition 9 A $3 D$ discrete line, denoted

$\mathcal{D}_{3 D}\left(a, b, c, \mu, \mu^{\prime}, e, e^{\prime}\right)$, with a main vector $(a, b, c)$ such that $(a, b, c) \in \mathbf{Z}^{3}$, and $a \geq b \geq c$ is defined as the set of points $(x, y, z)$ from $\mathbf{Z}^{3}$ verifying:

$$
\mathcal{D}\left\{\begin{array}{l}
\mu \leq c x-a z<\mu+e \\
\mu^{\prime} \leq b x-a y<\mu^{\prime}+e^{\prime}
\end{array}\right.
$$

with $\mu, \mu^{\prime}, e, e^{\prime} \in \mathbf{Z}$. $e$ and $e^{\prime}$ are called arithmetical width of $\mathcal{D}$.

The notion of $3 \mathrm{D}$ blurred segment (3D BS) is then proposed in [28] and it is shown that a $3 \mathrm{D}$ BS is bijectively projected into two projection planes.

Definition 10 Let $\mathcal{S}_{3 D}$ be a sequence of integer points in $\mathbf{Z}^{3}$. A set $\mathcal{S}_{3 D}$ is a $3 D$ blurred segment of width 
$\nu$ with a main vector $(a, b, c) \in \mathbf{Z}^{3}$ and $a \geq b \geq c$, if it possesses an optimal discrete line, denoted by

$\mathcal{D}_{3 D}\left(a, b, c, \mu, \mu^{\prime}, \omega, \omega^{\prime}\right)$ such that

- $\mathcal{D}(a, b, \mu, \omega)$ is optimal for the sequence of projections of points of $\mathcal{S}_{3 D}$ in the plane $(O, x, y)$ and $\frac{\omega-1}{\max (|a|,|b|)} \leq \mu$

- $\mathcal{D}\left(a, c, \mu^{\prime}, \omega^{\prime}\right)$ is optimal for the sequence of projections of points of $\mathcal{S}_{3 D}$ in the plane $(O, x, z)$ and $\frac{\omega^{\prime}-1}{\max (|a|,|c|)} \leq \mu^{\prime}$

Still in [28], the recognition algorithm of 3D MBS is given and has a linear complexity as in 2D. The main idea of the 3D MBS recognition is to add simultaneously the $2 \mathrm{D}$ points in the corresponding projection planes until at least one of them fails; i.e., we can not add any point more. Then, the 3D MBS is determined from the two corresponding MBS projected onto 2D planes. More details can be found in [28].

Similarly, the 3D ATC computation is performed by combining its two projections. Regarding the noise levels, we apply separately the noise estimation on each projection plane. Then, the noise level at each point of the $3 \mathrm{D}$ curve -without loss of generality- is the maximum value of the two projections. Note that the principle for the algorithm of 3D ATC remains the same as in 2D. Fig. 14 illustrates the 3D ATC of the 3D curves with and without noise.

\section{Conclusion and perspectives}

Based on the new notion of adaptive tangential cover [17], we have presented a new framework to analyze a noisy digital contour. More precisely, this approach combines the local scale analysis of a noise level esimator (in this paper most of the experimentations are done with the MT estimator) with the tangential cover and permits to obtain a geometric analysis which is adaptive to noise. A first new application was proposed with the classical problem of dominant point detection. The use of the ATC allows to obtain a parameter-free noiseresistant polygonalization method. Then, the ATC was used in different geometric estimators based on the tangential cover notion: (i) contour length estimator, (ii) tangent estimator with the $\lambda$-MST multigrid convergent tangent estimator and (iii) concave/convex part detector. Thanks to ATC, the robustness to noise of these estimators were improved without the need to manually adjust any parameter. An online demonstration based on the DGtal [29] and ImaGene [30] library can reproduce the main results of the ATC and polygonalization algorithm [31].

In this paper, we also proposed a first approach to extend the notion of ATC to 3D by using the noise levels of the $2 \mathrm{D}$ curves obtained by projecting the $3 \mathrm{D}$ curve. A complete study for 3D ATC would be investigated for any 3D curves. Another perspective is to study and to construct a local noise level estimator adapted to 3D curves.

Acknowledgment The authors would like to thank the referees for useful comments.

\section{References}

1. J.-P. Reveillès, Géométrie discrète, calculs en nombre entiers et algorithmique, thèse d'état. Université Louis Pasteur, Strasbourg (1991).

2. F. Feschet, L. Tougne, Optimal time computation of the tangent of a discrete curve: Application to the curvature. in: DGCI, Vol. 1568 of LNCS, 1999, pp. 31-40.

3. F. Feschet, Canonical representations of discrete curves, Pattern Anal. Appl. 8 (1-2) (2005) 84-94.

4. J. Lachaud, Digital shape analysis with maximal segments, in: Applications of Discrete Geometry and Mathematical Morphology - First International Workshop, WADGMM 2010, Istanbul, Turkey, 2010, pp. 14-27.

5. B. Kerautret, J.-O. Lachaud, Meaningful Scales Detection along Digital Contours for Unsupervised Local Noise Estimation, IEEE Transactions on Pattern Analysis and Machine Intelligence 34 (12) (2012) 2379-2392.

6. B. Kerautret, J.-O. Lachaud, Meaningful Scales Detection: an Unsupervised Noise Detection Algorithm for Digital Contours, Image Processing On Line 4 (2014) 98-115.

7. R. Malgouyres, F. Brunet, S. Fourey, Binomial Convolutions and Derivatives Estimations from Noisy Discretizations, in: Proceedings of the Int Conf on DGCI, Vol. 4992 of LNCS, Springer, 2008, pp. 370-379.

8. L. Cuel, J. Lachaud, Q. Mérigot, B. Thibert, Robust geometry estimation using the generalized voronoi covariance measure, SIAM J. Imaging Sciences 8 (2) (2015) 1293-1314. doi:10.1137/140977552.

URL http://dx.doi.org/10.1137/140977552

9. I. Debled-Rennesson, F. Feschet, J. Rouyer-Degli, Optimal blurred segments decomposition of noisy shapes in linear time, Computers \& Graphics 30 (1) (2006) 30-36.

10. M. Rodríguez, G. Largeteau-Skapin, E. Andres, Adaptive pixel resizing for multiscale recognition and reconstruction, in: Combinatorial Image Analysis, 13th International Workshop, IWCIA, Mexico, Vol. 5852 of LNCS, 2009, pp. 252-265.

11. A. Vacavant, T. Roussillon, B. Kerautret, J. Lachaud, A combined multi-scale/irregular algorithm for the vectorization of noisy digital contours, Computer Vision and Image Understanding 117 (4) (2013) 438-450.

12. T. P. Nguyen, I. Debled-Rennesson, On the local properties of digital curves, International Journal of Shape Modeling 14 (2) (2008) 105-125.

13. T. P. Nguyen, I. Debled-Rennesson, A discrete geometry approach for dominant point detection, Pattern Recognition 44 (1) (2011) 32-44.

14. P. Ngo, H. Nasser, I. Debled-Rennesson, Efficient dominant point detection based on discrete curve structure, in: International Workshop on Combinatorial Image Analysis IWCIA), Kolkata, India, November, Vol. 9448 of LNCS, 2015. 

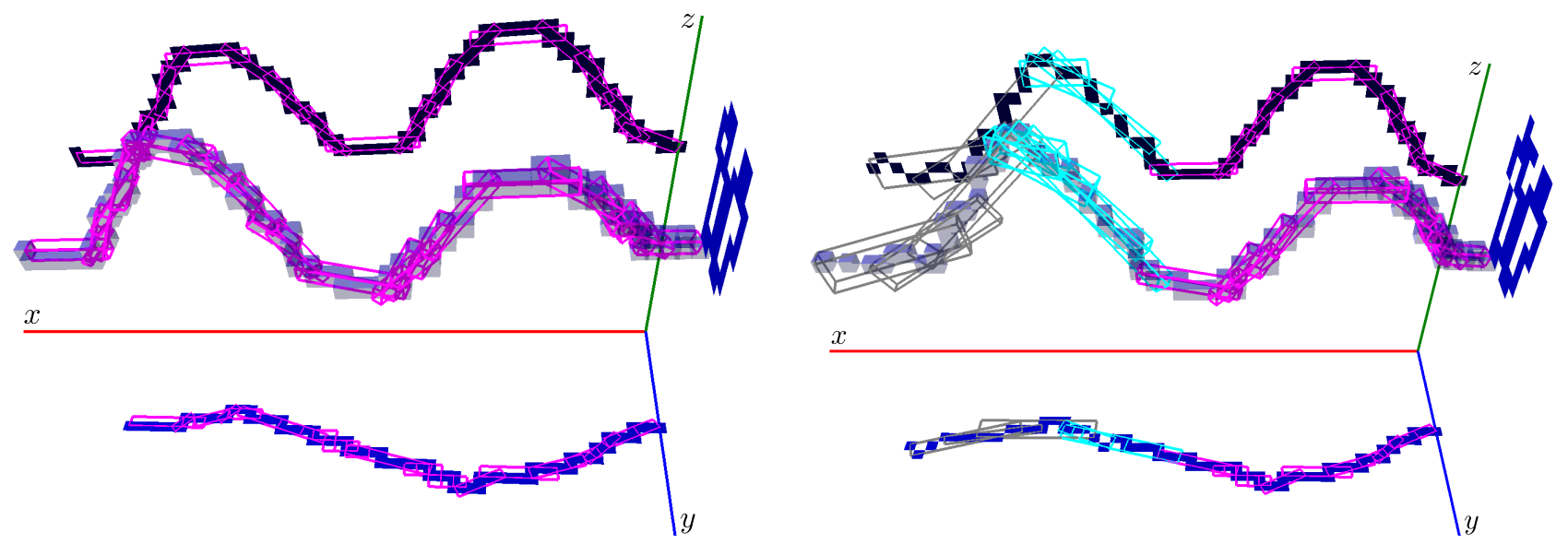

Fig. 14 Illustration of ATC in 3D, with MT estimator for the curve without noise (left) and with noise (right). In pink, blue and grey are segments associated to noise levels 1,2 and 3 respectively.

15. T. P. Nguyen, B. Kerautret, I. Debled-Rennesson, J. Lachaud, Unsupervised, fast and precise recognition of digital arcs in noisy images, in: Computer Vision and Graphics - International Conference, ICCVG 2010, Warsaw, Poland, September, Vol. 6374 of LNCS, 2010.

16. T. P. Nguyen, I. Debled-Rennesson, Decomposition of a curve into arcs and line segments based on dominant point detection, in: Image Analysis - 17th Scandinavian Conference, SCIA, Vol. 6688 of LNCS, 2011, pp. 794-805.

17. P. Ngo, H. Nasser, I. Debled-Rennesson, B. Kerautret, Adaptive tangential cover for noisy digital contours, in: Discrete Geometry for Computer Imagery - 19th IAPR International Conference, DGCI 2016, Nantes, France, Vol. 9647 of LNCS, 2016, pp. 439-451.

18. B. Kerautret, J.-O. Lachaud, M. Said, Meaningful Thickness Detection on Polygonal Curve, in: Proceedings of the 1st International Conference on Pattern Recognition Applications and Methods, SciTePress, 2012, pp. 372-379.

19. J.-O. Lachaud, A. Vialard, F. de Vieilleville, Fast, Accurate and Convergent Tangent Estimation on Digital Contours, Image and Vision Computing 25 (10) (2007) $1572-1587$.

20. J.-O. Lachaud, A. Vialard, F. de Vieilleville, Analysis and comparative evaluation of discrete tangent estimators, in: E. Andrs, G. Damiand, P. Lienhardt (Eds.), Proc. Int. Conf. Discrete Geometry for Computer Imagery (DGCI'2005), Poitiers, France, Vol. 3429 of LNCS, Springer, 2005, pp. 140-251.

21. A. Faure, L. Buzer, F. Feschet, Tangential cover for thick digital curves, Pattern Recognition 42 (10) (2009) 22792287.

22. D. Coeurjolly, B. Kerautret, J.-O. Lachaud, Extraction of Connected Region Boundary in Multidimensional Images, Image Processing On Line 4 (2014) 30-43.

23. T. Kanungo, Document Degradation Models and a Methodology for Degradation Model Validation, Ph.D. thesis, University of Washington (1996).

24. DGtalTools project: Tools associated with dgtal library. URL https://github.com/DGtal-team/DGtalTools

25. D. Coeurjolly, R. Klette, A comparative evaluation of length estimators of digital curves, IEEE transactions on pattern analysis and machine intelligence 26 (2) (2004) $252-258$.

26. H. Dörksen-Reiter, I. Debled-Rennesson, Convex and concave parts of digital curves, in: Geometric Properties for Incomplete Data, Computational Imaging and Vision, Springer, Vol. 31, 2006, pp. 145-160.

27. D. Coeurjolly, I. Debled-Rennesson, O. Teytaud, Segmentation and length estimation of 3d discrete curves, in: Digital and Image Geometry, Advanced Lectures [based on a winter school held at Dagstuhl Castle, Germany in December 2000], 2000, pp. 299-317.

28. T. P. Nguyen, I. Debled-Rennesson, On the local properties of digital curves, International Journal of Shape Modeling 14 (2) (2008) 105-125.

29. DGtal: Digital Geometry tools and algorithms library. URL http://libdgtal.org

30. Imagene, Generic digital Image library, http://gforge.liris.cnrs.frs/projects/imagene.

31. Adaptive tangential cover for noisy digital contours: Online demonstration, online, http://ipol-geometry. loria.fr/ kerautre/ipol_demo/ATC_IPOLDemo/. 